\title{
Chemical etching effects in porous silicon layers
}

\author{
D. Navarro-Urrios ${ }^{*}$ a,b, C. Pérez-Padrón ${ }^{\mathrm{a}}$, E. Lorenzo ${ }^{\mathrm{a}}$, N. E. Capuj ${ }^{\mathrm{a}}$, Z. Gaburro ${ }^{\mathrm{b}}$, C. J. Oton ${ }^{\mathrm{b}}$ and L. Pavesi $^{\mathrm{b}}$ \\ a Departamento de Física Básica, University of La Laguna, Avda. Astrofísico Fco. Sánchez, La Laguna, 38071 Spain \\ ${ }^{\mathrm{b}}$ INFM and Dipartimento di Física, University of Trento, Via Sommarive 14, Povo, Trento 38050 Italy
}

\begin{abstract}
We have studied the properties of $\mathrm{p}^{+}$-type doped porous silicon, formed by electrochemical etching, when is left in presence of the electrolyte for different post-etching times. Using an interferometric technique, we monitored the formation of the porous silicon layer during the electrochemical treatment as well as the change of its porosity during the post-etch process due to a chemical dissolution mechanism. These data are complemented with a study of the photoluminescence modification for different post-etching times.
\end{abstract}

Keywords: porous silicon, etching, electrochemistry, photoluminescence.

\section{INTRODUCTION}

Porous silicon (PS) is obtained by an electrochemical etch of silicon in an HF rich electrolyte. Its structure is complex with a random network of nanometer sized pores with large size dispersions. The nanostructured silicon which forms the skeleton of the material has fascinating properties which results mainly from quantum confinement of excited electron-hole pairs. Light emission under optical and electrical pumping in the visible and at room temperature has been observed in the last years [1,2]. Other applications of PS as a passive material have been demonstrated $[3,4,5,6]$. For instance, the modulation of the electrochemical current with time during the growth allows an easy realization of multilayer structures that can behave as distributed Bragg reflectors (DBRs) [7,8], microcavities [9], waveguides[10], etc.

The optical properties of PS are determined by both the complex network of nanometric air pores and by the silicon skeleton. Usual treatment assumes an effective medium approximation where the refractive index of PS is the results of some weighted average of the contributions of the air pores and of the crystalline skeleton. Therefore the optical properties of PS are very dependent on the details of its structure. Electrochemical etch of lightly doped (100)-oriented silicon produces a very branched structure of pores of very small diameter (less than $5 \mathrm{~nm}$ ), while heavily doped PS presents a geometry of bigger pores $(10$ to $50 \mathrm{~nm}$ ) parallel oriented [11]. In past works, it has been demonstrated that after the electrochemical fabrication of a PS layer, subsequent chemical etching in the HF electrolyte alters the properties of the layer due to enlargement of the diameter of the pores. Indeed, the light emission spectra of these postetched layers were blue shifted with respect to those of the original PS layers which supports an hypothesis of a reduction of the sizes of the silicon skeleton which in turn increases the quantum confinement of carriers and, hence, the energy of the emission $[1,12]$.

Using in-situ laser beam interference, the optical path of the PS layer $(n \times l$, where $n$ is the refractive index and $l$ the thickness) can be monitored during anodization [13]. Even two different beams with different incident angles can be analyzed simultaneously to obtain information about the porosity and etch rate drifts independently [14]. This kind of in-situ measurements have the advantage of allowing a continuous monitoring of the optical properties of the PS layer during the anodization. In this work we make use of this in-situ interferometric technique to study the etching in HF of a PS layer after the anodization process. We complement these data with photoluminescence (PL) measurements.

\footnotetext{
*navarro@science.unitn.it
} 


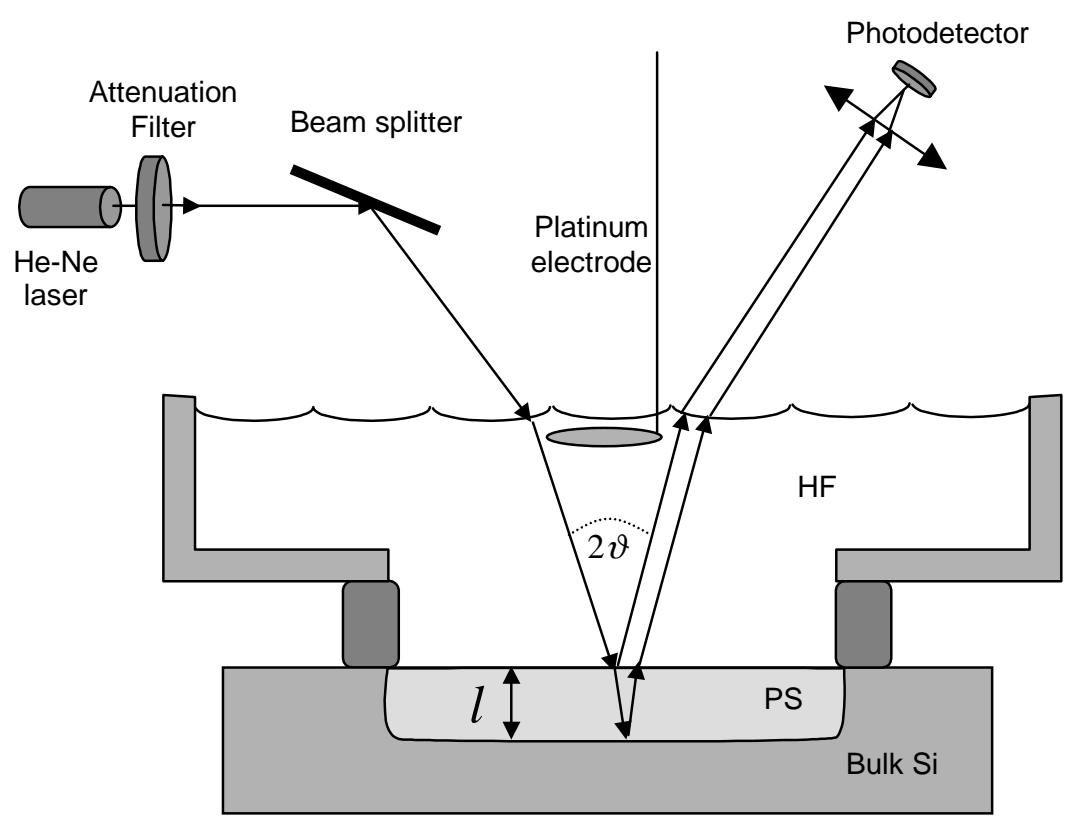

Figure 1. Sketch of the interferometry experimental setup. The reflected beams from the two interfaces are shown.

\section{THEORY}

Figure 1 sketches the experimental setup. The sample immersed in the electrolyte is illuminated by an extremely weak laser beam at an angle $\vartheta$. The reflectance of this beam is measured as a function of time during the electrochemical etching. The intensity of the reflected beam is a consequence of the interference of the two beams reflected at the various interfaces. In order to analyze the interference, a ray equation has to be solved [14]. Let us assume that a layer is being etched with a constant current density, $i$. e. we assume that the refractive index $n_{P S}$ of PS remains constant. It can be shown that the optical path difference $D$ between the beam reflected on the top PS/electrolyte interface and the one reflected on the bottom PS/silicon interface is:

$$
D=2 l \sqrt{n_{P S}^{2}-n_{H F}^{2} \sin ^{2}(\vartheta)}
$$

where $l$ is the PS thickness, $n_{H F}$ is the refractive index of the electrolyte, and $\vartheta$ is the incidence angle of the beam (see Fig. 1).

During the PS etching the current density is kept constant, therefore $n_{P S}$ remains constant while $l$ increases proportionally with time. As a consequence $D$ increases with time and the beam reflectance is an oscillating signal. The oscillation frequency $v_{c u r}(\mathrm{t})$ is linked to the change rate of the optical path difference $\frac{d D}{d t}$ :

$$
v_{c u r}(t)=\frac{1}{\lambda_{0}} \frac{d D}{d t}(t)
$$

where $\lambda_{0}$ is the wavelength of the beam. Therefore:

$$
v_{c u r}(t)=\frac{2}{\lambda_{0}} \sqrt{n_{p s}^{2}-n_{H F}^{2} \sin ^{2}(\vartheta)} \frac{d l}{d t}
$$


In this work, we are interested in the variation of PS when exposed to the electrolyte after the electrochemical attack. For this reason we leave the PS in the solution after the current has been turned off, and we look at the evolution of the reflectance signal. An oscillating signal is measured also in this case. This means that during the post-etching treatment the density of the PS layer is reduced by the chemical etching of HF. Equations (1) and (2) are still valid. However the optical path difference $D$ changes are mainly associated with reductions in the PS layer refractive index $n_{P S}$, while $l$ is constant (see later). The oscillating frequency of the reflectance during the post-etching process $v_{p e}(t)$ follows from (2) when only $n_{P S}$ varies with time:

$$
v_{p e}(t)=\frac{2 l}{\lambda_{0}} \frac{n_{P S}(t)}{\sqrt{n_{P S}^{2}-n_{H F}^{2} \sin ^{2}(\vartheta)}} \frac{d n_{P S}}{d t}
$$

As $n_{P S}>n_{H F}$ one can expand (4) in terms of $x=\frac{n_{H F}^{2} \sin ^{2}(\vartheta)}{n_{P S}^{2}}$ and obtains:

$$
v_{p e}(t) \cong \frac{2 l}{\lambda_{0}} \frac{d n_{P S}}{d t}\left\{1+\frac{n_{H F}^{2} \sin ^{2}(\vartheta)}{n_{P S}^{2}}+O\left(x^{2}\right)\right\}
$$

For $\vartheta<10^{\circ}$ :

$$
v_{p e}(t) \cong \frac{2 l}{\lambda_{0}} \frac{d n_{P S}}{d t}
$$

Equation (6) relates the oscillating reflectance frequency with the variation in the PS refractive index.

\section{EXPERIMENT}

The porous silicon samples were fabricated starting from $\mathrm{p}^{+}$-type boron-doped silicon substrates of low resistivity $(0.01$ $\Omega \mathrm{cm})$. The electrochemical etch was performed at room temperature with an electrolyte obtained by mixing a $31 \%$ volumetric fraction of aqueous HF (48 wt. \%) with ethanol. We performed the electrochemical etch in dark applying a constant current density of $40 \mathrm{~mA} / \mathrm{cm}^{2}$ on an area of $1 \mathrm{~cm}^{2}$. The HP $6612 \mathrm{C}$ current source was controlled by a computer.

Once the electrochemical etch was ended, the PS layer was left in the electrolyte in dark, and the post-etch chemical etching was initiated. Several post-etching duration times were used. For photoluminescence measurements the PS layers were extracted from the electrolyte and dried in air.

The optical set up of the experiment is shown in Fig. 1. The in situ monitoring of the optical path change was performed using a $632.8 \mathrm{~nm}$ He-Ne laser with a power of $1 \mu \mathrm{W}$. This low power level assures no effect on the electrochemical etch and post etching due to the light beam. The incidence angle of the laser beam on the sample was $8^{\circ}$ and the spot size was $\sim 2 \mathrm{~mm}$. The reflected beam was focalized onto a silicon photodiode. The current signal of the photodiode was amplified and acquired with a computer.

The photoluminescence (PL) experiments were performed pumping with all the lines of an Ar CW laser, focalizing a $100 \mathrm{~mW}$ beam on the sample, and collecting with a $25 \mathrm{~cm}$ focal length monochromator, and finally detecting the signal with a photomultiplier. Since the intensities of the PL spectra for the various samples were not comparable, the PL data we are reporting are normalized to their maximum. 


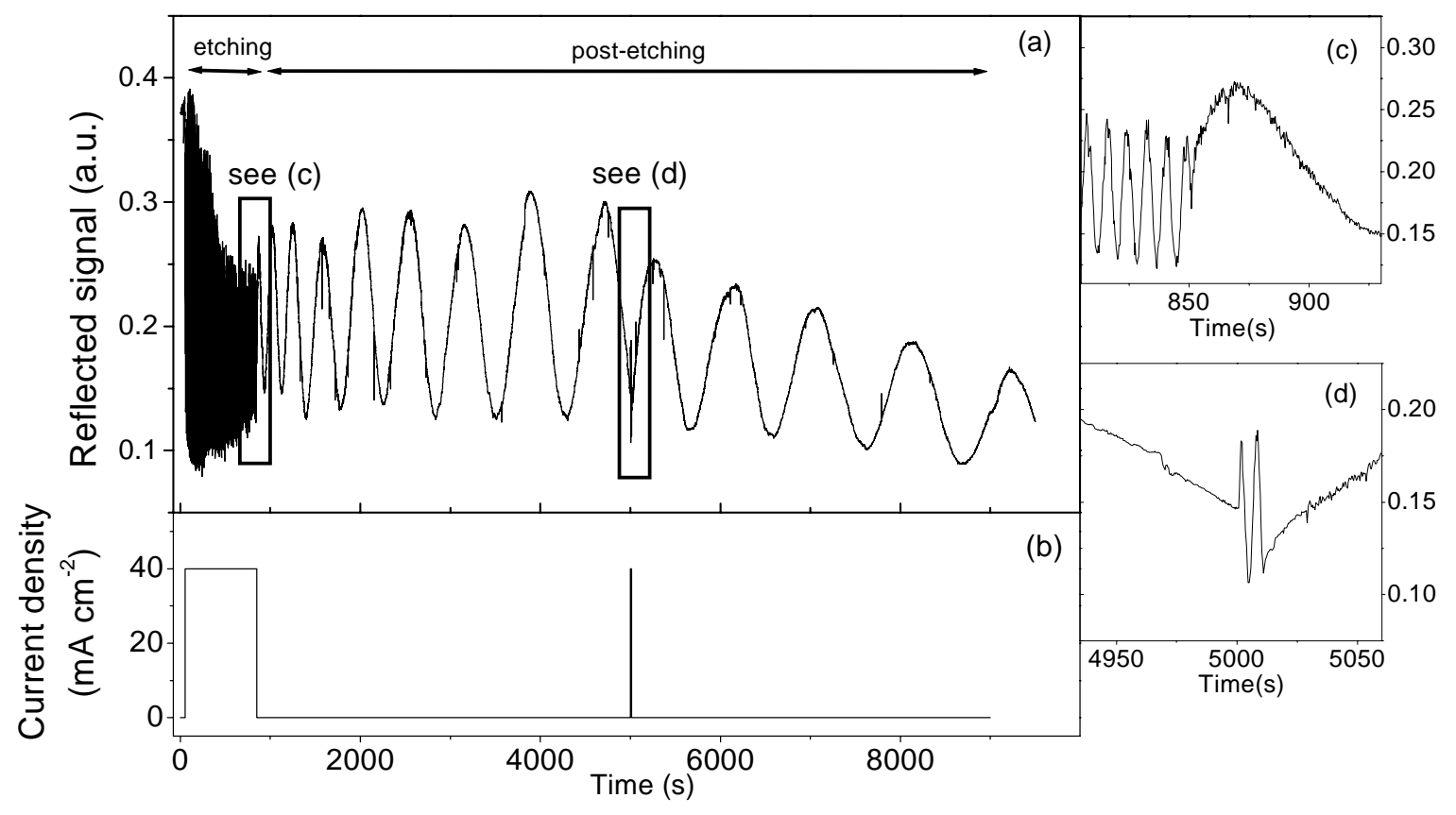

Fig. 2: (a) Reflected signal during the anodization and the post-etching processes. (b) Electrochemical current density applied versus time (800 s of anodization time and a short pulse after $4150 \mathrm{~s}$ of post-etching). (c) and (d): Blow up of the reflected signal near the current changes. The frequency and the phase sign changes can be clearly observed.

\section{RESULTS}

We made PS samples with different thicknesses, i. e. electrochemical etch times (400, 800, 1200 and $1600 \mathrm{~s})$, and we analyzed the post etching for much longer times (up to $18000 \mathrm{~s}$ ). A representative example of the reflected signal during the etching and the post-etching periods is reported in Fig. 2. Some spikes appear in the reflected intensity mainly due to the gas bubbles which form during the etching. An oscillating reflectance is observed as theoretically expected. The fast oscillations during the electrochemical etch are due to the rapid increase of the PS thickness. When the electrochemical attack is ended, the reflectance signal is still oscillating though with a much smaller velocity (see the zoom in Fig. 2c). This is somehow unexpected and indicates that some kind of etching is occurring even when the current is stopped. Apart from the frequency changes, it is also worth noticing in Fig. 2c that the phase of the oscillating reflectance signal changes sign. Since during the electrochemical etch the optical path of the layer grows, a change of phase when the current is stopped indicates that the optical path starts to decrease. This can be explained by a decrease of the refractive index of the PS layer due to the chemical etching of the silicon. It is also interesting the fact that the frequency of the reflectance oscillations decreases during the post-etching process.

After $4150 \mathrm{~s}$ of chemical etching, i. e. at time $5000 \mathrm{~s}$, a $10 \mathrm{~s}$ short current pulse was applied to increase the PS layer thickness. During this short electrochemical etching step, the reflectance signal starts again to oscillate with high frequency (see Fig. 2d). After the current pulse, the reflectance signal continues to oscillate with the same frequency and phase it had before. This simple experiment is very indicative. In fact, one could attribute the low frequency reflectance oscillations observed during the post-etching treatment to some slow variations of the electrolyte composition within the PS layer, due to the out-diffusion of some chemical components (e.g. $\mathrm{F}^{-}$ions). This diffusion 
could change the effective refractive index of the PS layer producing a change in its optical thickness which could be observed by variations in the reflectance. The experiment shown in Fig. 2d discards this explanation. The short current pulse recovers the electrolyte composition within the PS layer and starts again the electrochemical etch of PS. After the small pulse of current the frequency of the reflectance signal is the same as that before the current pulse. This fact demonstrates that the slow oscillations during the post-etching are not a transient that occurs after any current stop due to out-diffusion of chemical species from the PS, but are due to a chemical process that changes the optical properties of the PS layer.
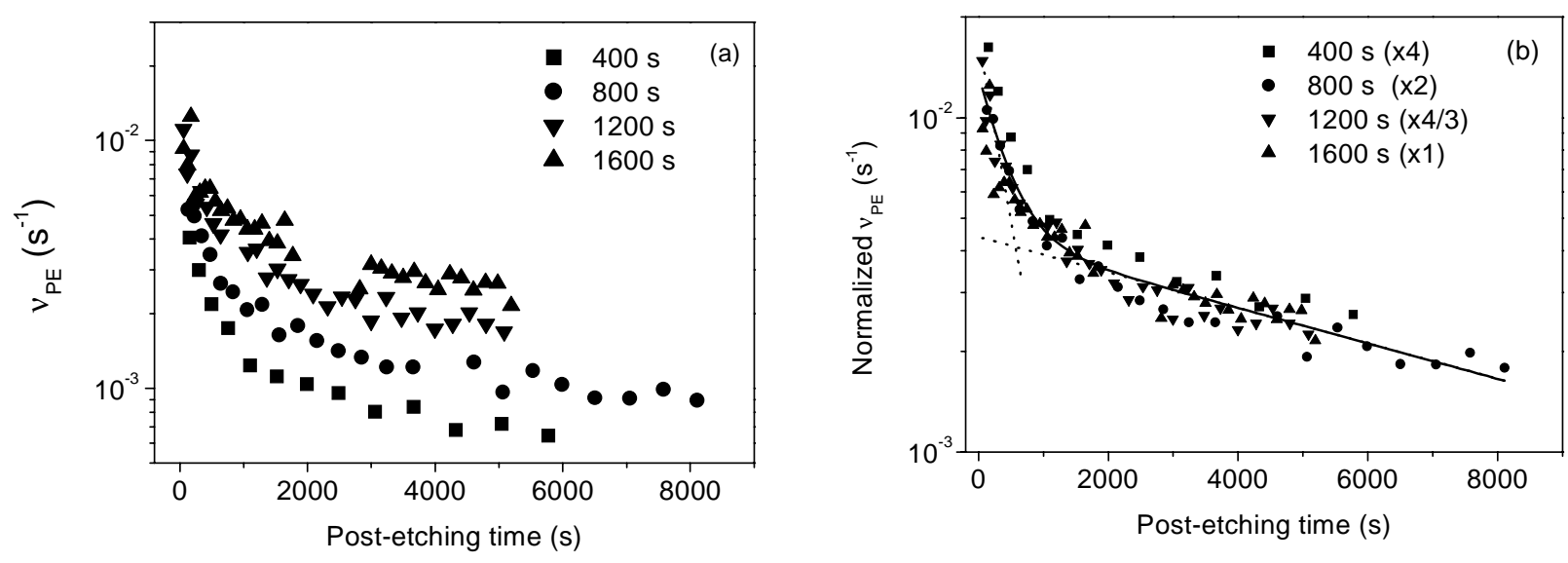

Fig 3: (a) Frequency of the oscillating reflected signal during the post-etching treatment of four different PS samples produced with the same current but with various electrochemical etch times (squares: 400 s, dots: 800 s, downward triangles: 1200 s, upward triangles: 1600 s). (b) Normalized frequency vs the post-etching time. The normalized frequency is obtained by multiplying the frequency by $1600 \mathrm{~s}$ and dividing by the corresponding electrochemical etch time. The various scaling factors are reported in the figure within parenthesis. The symbols refer to the same sample as in (a). The line is a two exponential fit to the data. The two exponentials are shown as dotted lines.

From the reflectance oscillations, the time dependence of their frequencies can be extracted. Figure 3a shows the frequency of the signal versus the post-etching time for four different PS samples obtained with the same current but different length of the electrochemical attack: 400, 800, 1200 and 1600 s. Since eq. (6) predicts a frequency of oscillations proportional to the thickness of the layer, in fig. $3 \mathrm{~b}$ the thickness normalized frequencies versus the postetching time are reported for the various PS samples. All the data points align which supports the theoretical model we used and implies that the rate of refractive index variation is the same for the different PS layer thickness. The data can be fitted with a double exponential with $\tau_{1}=400 \mathrm{~s}$ and $\tau_{2}=8200 \mathrm{~s}$ (lines in Fig. 3b). 

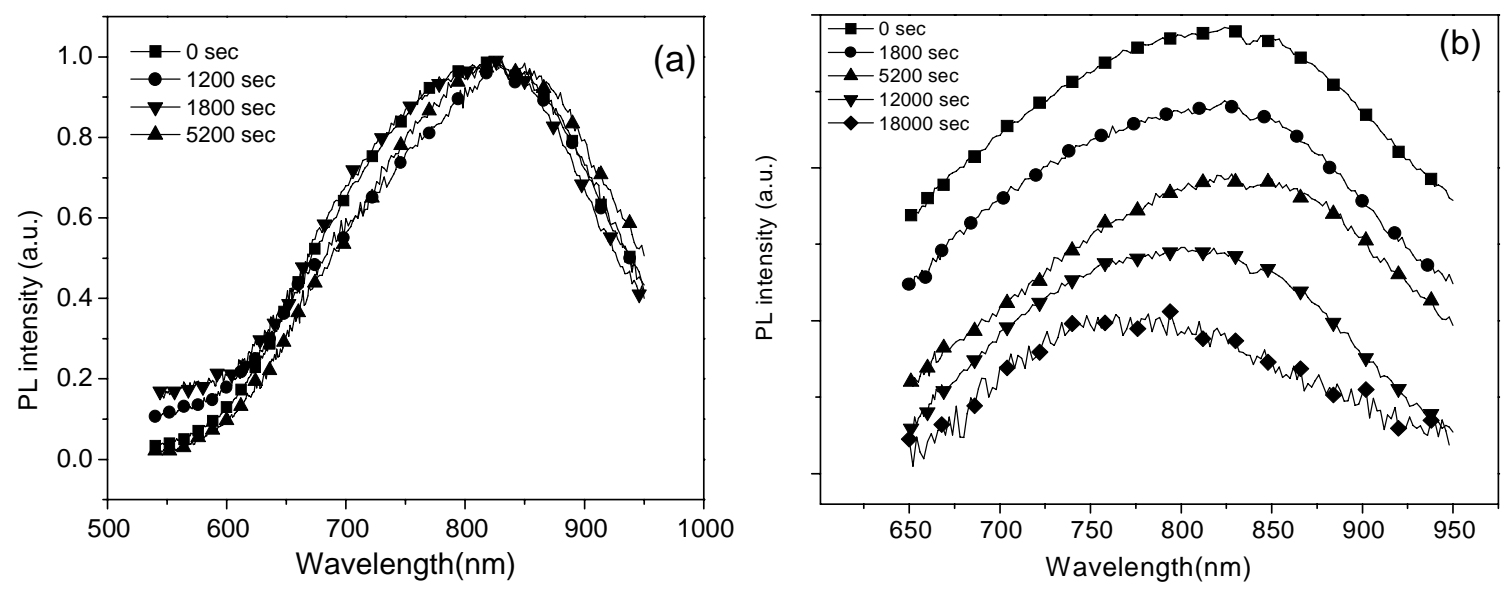

Fig. 4: (a) PL spectra for short post-etching time samples. (b) Waterfall plot of PL curves for long post-etching time samples.

In fig. 4 we show the PL measurements for various PS samples obtained with an electrochemical etching $800 \mathrm{~s}$ long but with different post-etching durations. In the first minutes of the post-etching treatments (Fig. 4a), a slight luminescence lineshape change is observed where the relative intensity of the short wavelength (550-700 nm) emission increases until a maximum is reached at $1800 \mathrm{~s}$ of post-etching time. For longer times, this short wavelength contribution decreases. For even greater post-etching times (Fig. 4b), a blue shift of the emission is appreciated. The peak position of the luminescence versus post-etching time is shown in fig. 5 .

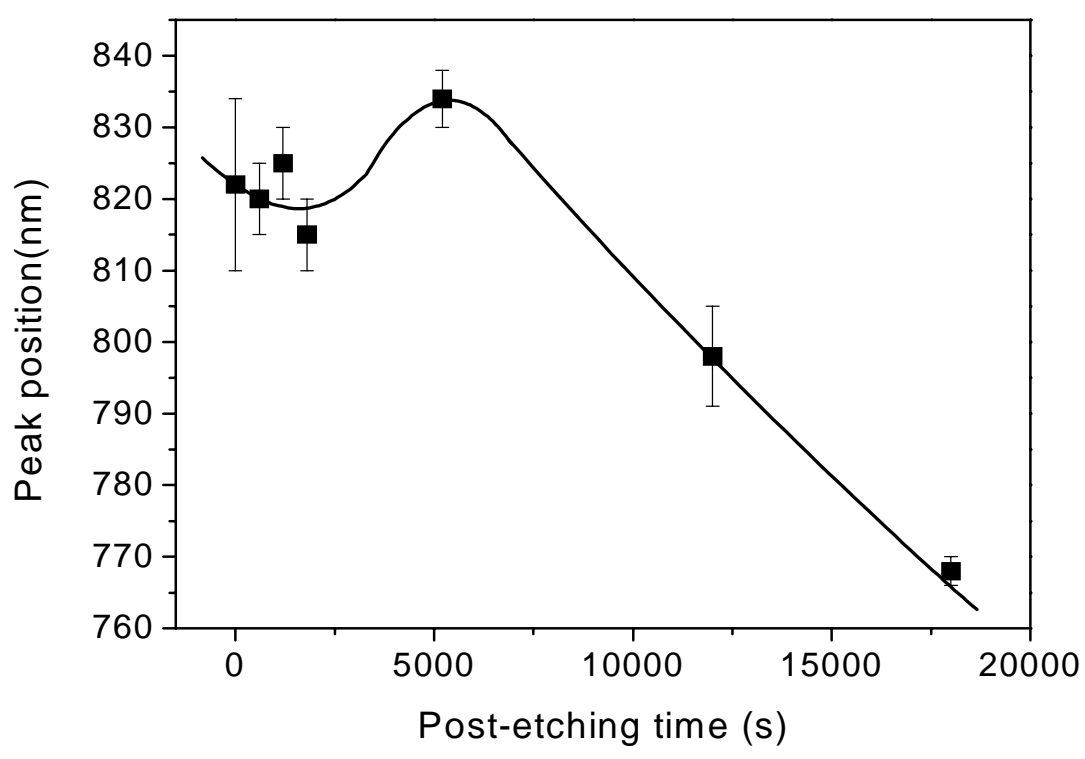

Fig. 5: PL peak position versus post-etching time. The solid line is a guide for the eye. 


\section{DISCUSSION}

From the experimental study we have found that:

1. during the post-etching the refractive index of PS decreases following a slow chemical etch of the layer;

2. the rate of the decrease of the refractive index is characterized by two time scales: at short times a fast decrease in $n_{P S}$ is observed and at long times a slower decrease in $n_{P S}$ is observed;

3. the luminescence spectra of PS depends on the post-etching time;

4. a contribution at short wavelength in the emission appears and disappears at short times;

5. the luminescence blue shifts at longer times.

We propose a model that could explain the behaviors observed in PL as well as the in situ monitoring of the postetching. It is based on the very inhomogeneous structure of the silicon skeleton in PS. It is well known that PS formed on $\mathrm{p}^{+}$-type silicon is mainly composed by a large (3-10 $\mathrm{nm}$ in size) highly filamentary crystalline silicon network. These filaments are coated by smaller (1-4 nm in size) silicon nanostructures. These two different kinds of silicon nanostructures emit light in two different wavelength ranges because of the different quantum confinement characteristics. In addition, they are chemically etched by HF at different rates because of the different surface curvature and surface-volume ratio. Considering the inhomogeneous nature of PS we could explain our experimental observations as follows. The residence of the PS layer in the electrolyte causes a slow chemical etching of PS with a negligible PS thickness increases but a significant increase of porosity, $i$. e. decrease of refractive index, because silicon is consumed during the etch. The first fast exponential decrease of the refractive index is due to the etching of the small silicon nanocrystals. At the same time the short wavelength component of the luminescence emission of PS increases and then decreases following the rapid formation of small silicon nanocrystals and then their complete dissolution. At longer post-etching times, a second process becomes dominant characterized by a different rate. This process is the dissolution of the large filamentary silicon skeleton. The dissolution rate is now slower due to the smaller surface-volume ratio and the longer surface curvature radii. The luminescence emission band shifts to the blue following the progressive thinning of the filamentary silicon nanostructures.

\section{CONCLUSIONS}

In this work we have investigated the structural changes of $\mathrm{p}^{+}$-type PS when chemically etched in HF for long times. We have characterized the etching process in terms of the change rate of the optical path making use of an interferometric setup. Two rates have been observed: one fast and one slow. The structure modification with the postetching time leads also to appreciable variations in the photoluminescence spectra. Besides the main gaussian centered at $800 \mathrm{~nm}$ we observed a short wavelength contribution that grows and disappears in the first minutes of the postetching treatment, followed by a blue shift. We suggest an explanation for this behavior in terms of two different scales of silicon nanostructures that are etched at different velocities.

\section{ACKNOWLEDGEMENTS}

This work has been supported by the Science and Technology Ministry of Spain (MCYT) (Project No. MAT 200200044), the Canary Islands Government (Project No. PI2001/093, PI2001/074), the European project EC-SINERGIA and the Italian project SMOG-INFM. We thank I. Martín for the photoluminescence measurements and stimulating discussions. 


\section{REFERENCES}

1 L. T. Canham, Appl. Phys. Lett. 57 (10), 1046 (1990).

2 A. Halimaoui, C. Oules, G. Bomchil, A. Bsiesy, F. Gaspard, R. Herino, M. Ligeon and F. Muller, Appl. Phys. Lett. 59304 (1991).

3 M. J. Sailor, in Properties of Porous Silicon, ed. L. T. Canham IEE Inspec - London U. K., 1997, p.364.

4 W. Theiß, Surf. Sci. Rep. 29 (3/4), 91 (1997).

5 M.V. Wolkin, S. Chan and P.M. Fauchet, Phys. Stat. Sol. (a) 182,573 (2000)

6 W. Liu et al. J. Vac. Sci. \& Techn. B 21, 168 (2003)

7 M. G. Berger, R. Arens-Fischer, M. Kruger, S. Billat, H. Luth, S. Hilbrich, W. Thei $\beta$ and P. Grosse, Thin Solid Films 297, 137 (1997).

8 V. Agarwal and J. A. del Rio, Appl. Phys. Lett, 82,1512 (2003)

9 L. Pavesi, Riv. Nuovo Cimento 20, 1 (1997).

10 S. Nagata, C. Domoto, T. Nishimura and K. Iwameji, Appl. Phys. Lett. 72, 2945 (1998).

11 G. Bomchil, A. Halimaoui, I. Sagnes, P. A. Badoz, I. Berbezier, P. Perret, B. Lambert, G. Vincent, L. Garchery and J. L. Regolini, Appl. Surf. Sci. 65/66, pp. 394-407 (1993).

12 M. B. Robinson, A. C. Dillon and S. M. George, Appl. Phys. Lett. 62 (13) p. 1493 (1993).

13 M. Thönissen, M. G. Berger, S. Billat et al. Thin Solid Films 297, 92 (1997).

14 Z. Gaburro, C. J. Oton, P. Bettotti, L. Dal Negro, G. Vijaya Prakash, M. Cazzanelli and L. Pavesi, to be published in J. Electrochem. Soc. 